\title{
Prediction Approach of Critical Node Based on Multiple Attribute Decision Making for Opportunistic Sensor Networks
}

\author{
Qifan Chen, ${ }^{1,2}$ Linlan Liu,, ${ }^{1,3}$ Zhiyong Yang, ${ }^{1,2}$ and Kai Guo ${ }^{1,3}$ \\ ${ }^{1}$ Internet of Things Technology Institute, Nanchang Hangkong University, Nanchang 330063, China \\ ${ }^{2}$ School of Software, Nanchang Hangkong University, Nanchang 330063, China \\ ${ }^{3}$ School of Information Engineering, Nanchang Hangkong University, Nanchang 330063, China
}

Correspondence should be addressed to Linlan Liu; liulinlan@nchu.edu.cn

Received 14 January 2016; Accepted 29 March 2016

Academic Editor: Fei Yu

Copyright (C) 2016 Qifan Chen et al. This is an open access article distributed under the Creative Commons Attribution License, which permits unrestricted use, distribution, and reproduction in any medium, provided the original work is properly cited.

\begin{abstract}
Predicting critical nodes of Opportunistic Sensor Network (OSN) can help us not only to improve network performance but also to decrease the cost in network maintenance. However, existing ways of predicting critical nodes in static network are not suitable for OSN. In this paper, the conceptions of critical nodes, region contribution, and cut-vertex in multiregion OSN are defined. We propose an approach to predict critical node for OSN, which is based on multiple attribute decision making (MADM). It takes RC to present the dependence of regions on Ferry nodes. TOPSIS algorithm is employed to find out Ferry node with maximum comprehensive contribution, which is a critical node. The experimental results show that, in different scenarios, this approach can predict the critical nodes of OSN better.
\end{abstract}

\section{Introduction}

In Opportunistic Sensor Network (OSN), the critical nodes are very important to keep normal operation of networks. In practical applications, if the critical nodes can be predicted, the network could be optimized according to the attributes of critical nodes, which helps improving the robustness of the network. In network maintenance, maintainers can focus on monitoring the status of critical nodes so that the failures of the network could be resolved immediately, which can dramatically reduce the time and the cost of network maintenance. Therefore, predicting critical nodes of OSN has great significance.

\section{Related Work}

OSN is a kind of Wireless Sensor Networks. It perceives the surrounding environment by sensor nodes and transports messages by the meeting opportunities of Ferry nodes. Hence, it has the characteristics of Mobile Opportunity Network [1] and Wireless Sensor Network [2]. The current study of OSN critical nodes is very little. Nevertheless, in some related fields, researchers have made some progress such as node importance evaluation [3-7] and network cutvertex judgement [8-14].

Corley and Sha [15] proposed that the critical nodes in a weighted network are those whose removal from the network results in the greatest increase in shortest distance between two specified nodes. This method could be applied to estimate the end-to-end nodes. However, it is powerless to estimate the critical nodes in the whole network. Chen et al. [16] studied a method to estimate the relative importance of nodes by comparing the number of spanning trees. Although this method could estimate the critical nodes of the whole network, it has the problem of high computing complexity. So it is not suitable in practical applications. In resistance network, Xiao et al. [17] did the research on the energy consumption model to evaluate the importance degree of nodes. This method estimates the critical node by comparing the increase of the average energy consumption of the network after the nodes are removed. Goyal and Caffery [18] discussed the split of ad hoc networks. They utilized network survivability concepts to detect the critical links in an ad hoc wireless network. This method is based on the precondition 


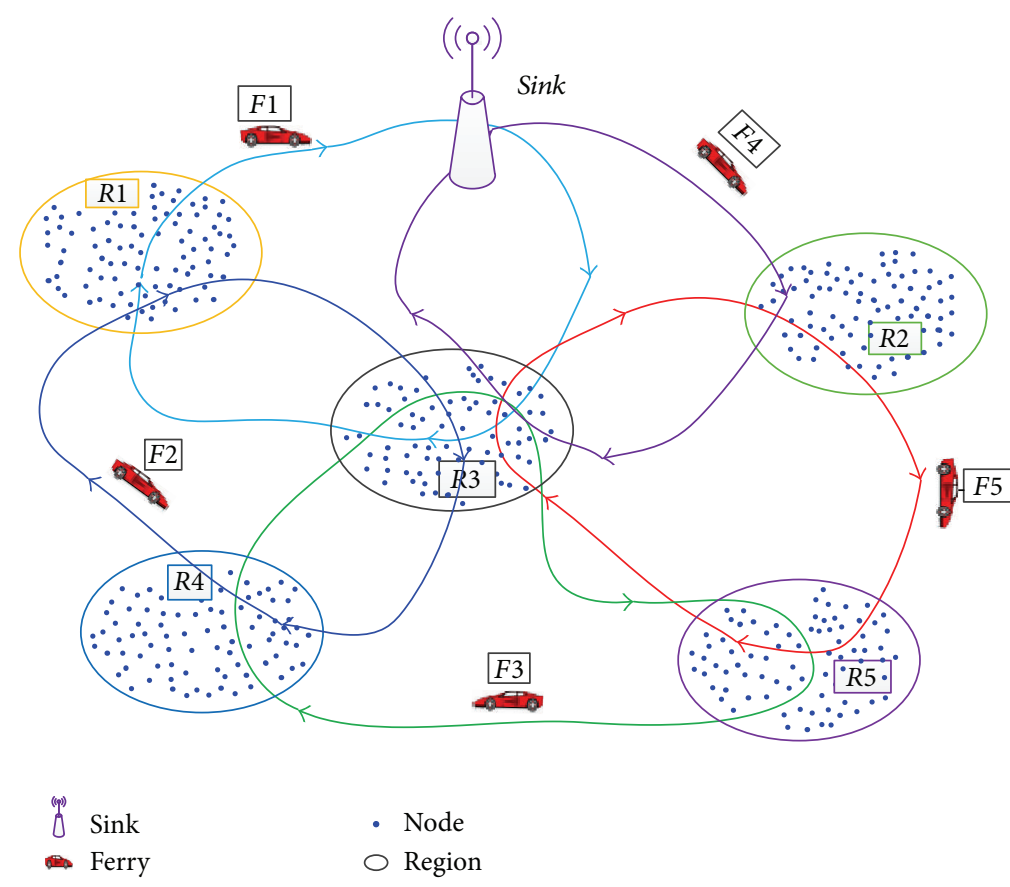

(a) Scenario

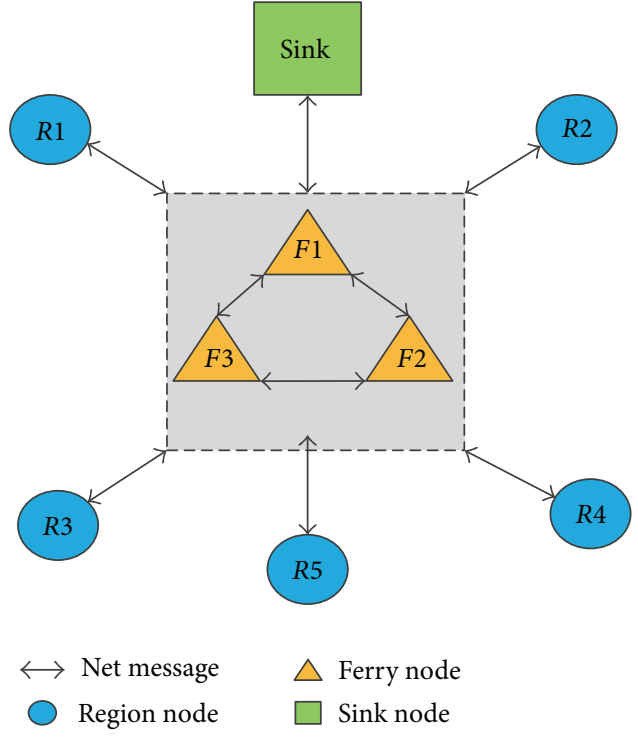

(b) Scenario model

FIGURE 1: OSN scenario.

that the nodes can locate themselves, which has great application limitations.

With the research above, it is always one-sided to evaluate the network through a single evaluation index. Considering the influence of node degree, node closeness, node betweenness, equivalent topology, and neighbor lists, Hu et al. [19] conducted experiments with three real typical networks to show that their method is more accurate than using a single evaluation index. Liu et al. [20] proposed a method to estimate critical nodes by combining the residual lifetime of nodes and the network energy consumption. Due to the shortcomings of the methods of node deletion and node contraction, Zhou et al. [21] exploited the evaluation matrix of node efficiency and node importance to determine the critical nodes in complex network. This method reflects the significance of divergences between two nodes and can evaluate the importance exactly. In a similar way, Fan and Liu [22] discussed the local and global importance of nodes and presented an evaluation method based on transfer efficiency matrix which takes not only the interactions between adjacent nodes but also the nonadjacent nodes' contributions into account, thus obtaining a more accurate node importance evaluation result.

As a dynamic network, current static prediction methods of critical nodes are not appropriate for OSN. Depending upon the researches above, in this paper, the stage contribution and the region contribution are proposed to evaluate the node importance. Then an algorithm is designed which is based on the multiple attribute decision making (MADM) to predict the critical nodes of OSN.

\section{Scenario Model and Definitions}

3.1. Scenario Model. The monitoring areas of application scenarios like environmental monitoring are very large. Therefore, the maintainers tend to monitor the key regions instead of the whole network. In OSN, the messages of the network are collected through the communication opportunities supported by mobile nodes. As shown in Figure 1(a), our research is proposed for the OSN with multiple regions and the nodes in the regions are fixed. There are Ferry nodes between regions supporting communication opportunities to Sink nodes and the trajectory of Ferry nodes could be a specific way or a random way.

In Figure 1(b), $R 1 \sim R 5$ are region nodes; $F 1 \sim F 3$ are Ferry nodes. The region nodes and Sink nodes are fixed and isolated. Those two kinds of nodes cannot communicate with each other without Ferry nodes. The Ferry nodes can move among regions, carrying and transporting messages between Sink nodes and other regions.

In this paper, the following assumptions are made:

(1) In our research, each region is abstracted as a "super node" called region node.

(2) Regardless of the Ferry nodes' memory, it is assumed that Ferry node can collect all the messages from each node it meets.

(3) Regions and Ferry nodes and Sink nodes in the network have unique identity information.

(4) The network has a time synchronization mechanism. 


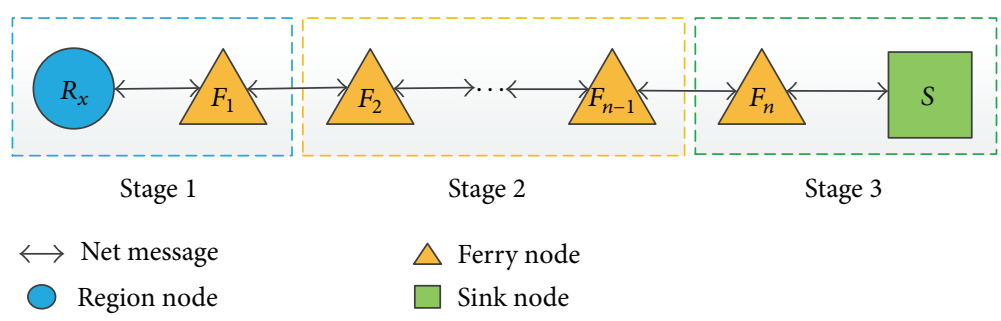

FIGURE 2: OSN message transmission.

In Figure 1, Ferry nodes in the whole network play a role of transport medium so that the Ferry nodes are the key to ensure the connectivity of the whole network. Besides, as a kind of "super node," the region nodes consist of a group of sensor nodes. Individual sensor node's failure in regions will have little impact on the whole network. Therefore, we can learn from the research above that the critical node of OSN could be a Ferry node.

3.2. Definition of Critical Nodes. Define Opportunistic Sensor Networks as $G$. Define $F$ as a critical node of $G$ when the possibility of network split caused by the removal of node $F$ is the largest. According to the definition above, when the cut-vertices of OSN are removed, the network will be divided. Therefore, we can learn that the cut-vertices of OSN must be critical nodes.

OSN is a dynamic network which transfers data by the "Store-Carry-Forward" mechanism. The traditional parameters such as node degree [23,24], node betweenness [25-29], node closeness [30], aggregation [31, 32], and other network parameters are not suitable for OSN. Therefore, it is necessary to set up an appropriate evaluation index for OSN.

In OSN, Ferry nodes are transport mediums. So their job is to transport messages between Sink nodes and region nodes. In order to accurately estimate each Ferry node's importance to the network, the effect of Ferry nodes on OSN must be considered properly. With intensive analysis of OSN's routing mechanism, the region messages' life cycle can be divided into three stages as shown in Figure 2.

In the first stage, Ferry nodes receive network messages from regions and then carry them out. In the second stage, the network messages will be forwarded among Ferry nodes. At last, Ferry nodes transport messages to the Sink nodes. These three stages can not only depict the message propagation of OSN clearly but also show the important role of Ferry nodes obviously.

3.3. Definition of First Stage Contribution. Define a time slice as $T$ and the Sink node receives $M_{j}$ pieces of messages from region $R_{j}$ in $T$. If the Ferry node $F_{i}$ has forwarded messages in the first stage, the total number of the forwarding messages is $n_{i j}\left(n_{i j} \leq M_{j}\right)$. Define the First Stage Contribution (FSC) of node $F_{i}$ to region $R_{j}$ as $n_{i j} / M_{j}$, denoted by $\operatorname{FSC}\left(F_{i}, R_{j}\right)$.

3.4. Definition of Second Stage Contribution. Define a time slice as $T$ and the Sink node receives $M_{j}$ pieces of messages from region $R_{j}$ in $T$. If the Ferry node $F_{i}$ has forwarded messages in the second stage, the total number of the forwarding messages is $m_{i j}\left(m_{i j} \leq M_{j}\right)$. Define the Second Stage Contribution (SSC) of node $F_{i}$ to region $R_{j}$ as $m_{i j} / M_{j}$, denoted by $\operatorname{SSC}\left(F_{i}, R_{j}\right)$.

3.5. Definition of Third Stage Contribution. Define a time slice as $T$ and the Sink node receives $M_{j}$ pieces of messages from region $R_{j}$ in $T$. If the Ferry node $F_{i}$ has forwarded messages in the third stage, the total number of the forwarding messages is $k_{i j}\left(k_{i j} \leq M_{j}\right)$. Define the Third Stage Contribution (TSC) of node $F_{i}$ to region $R_{j}$ as $k_{i j} / M_{j}$, denoted by $\operatorname{TSC}\left(F_{i}, R_{j}\right)$.

3.6. Definition of Region Contribution. Define a time slice as $T$. The stage contributions of Ferry node $F_{i}$ to region $R_{j}$ are $\operatorname{FSC}\left(F_{i}, R_{j}\right), \operatorname{SSC}\left(F_{i}, R_{j}\right)$, and $\operatorname{TSC}\left(F_{i}, R_{j}\right)$. Define region contribution (RC) of node $F_{i}$ to region $R_{j}$ as $\mathrm{RC}\left(F_{i}, R_{j}\right)=$ $\operatorname{FSC}\left(F_{i}, R_{j}\right)+\operatorname{SSC}\left(F_{i}, R_{j}\right)+\operatorname{TSC}\left(F_{i}, R_{j}\right)$, denoted by RC $\left(F_{i}, R_{j}\right)$.

The region contribution can reflect both the Ferry nodes' contributions to regions and the dependence of the regions on Ferry nodes. It means that the bigger region contribution the node has, the higher possibility leading to the network split the node possesses and the node is more likely to be a critical node. If the region contribution from node $F_{x}$ to region $R_{y}$ equals 1 , it means that region $R_{y}$ is fully dependent on node $F_{x}$; that is to say, if node $F_{x}$ is removed, region $R_{y}$ will be isolated from the whole network.

According to the researches above, we can infer that the node is a cut-vertex of the network when the region contribution equals 1 and it must be a critical node.

\section{MADM Based Prediction Method of Critical Node of OSN}

According to the research mentioned above, the critical node prediction method for OSN can be described as the following steps.

Step 1. Calculate each Ferry node's region contributions in order to determine whether the network has cut-vertexes or not. If there are no cut-vertexes in the network, go to Step 2.

Step 2. Find out a node which most likely leads to the network split and it must be a critical node. The region contribution shows the dependence of regions on Ferry nodes. We can learn that the higher region contribution the node has, the higher risk of network split it will have. Based on the theory 
above, we first take each Ferry node as a single evaluation scheme. Then, the TOPSIS method is applied to evaluate the comprehensive region contribution of Ferry nodes.

4.1. Algorithm Description. It is meaningless to predict such a dynamic network like OSN by calculating region contributions within a single time slice such as $\Delta T$. However, a single prediction result can be defined as a suspected critical node. After setting $N \cdot \Delta T$ as the total time length of prediction, $N$ suspected critical nodes will be found. Then, the frequency of each node to be estimated as a suspected critical node can be recorded.

We assume an OSN with $d$ Ferry nodes. Each node may lead to network split. If node $F_{i}$ has been estimated as a suspected critical node $q$ times, the appearance probability of node $F_{i}$ could be figured out as $P_{s}\left(F_{i}\right)$ :

$$
P_{s}\left(F_{i}\right)=\frac{q}{N\left(c_{d}^{1}+c_{d}^{1} c_{d-1}^{1}+c_{d}^{1} c_{d-1}^{2}+\cdots+c_{d}^{1} c_{d-1}^{d-1}\right)} .
$$

Denote the maximum of $P_{s}\left(F_{i}\right)$ as $\max \left(P_{s}\right)$ and the corresponding node as $F_{k}, k \in\{1,2,3, \ldots, d\}$. We define this node as a predicted critical node.

$\Delta T$ is a single time slice of the network. The details of $\Delta T$ are defined as follows: it is assumed that there are $n$ Ferry nodes to be determined in the network so that the corresponding solution sets can be denoted by $F=$ $\left(F_{1}, F_{2}, \ldots, F_{n}\right)$ and there are $m$ regions in the network so the region contribution of each Ferry node can be denoted by an attribute set $S=\left(S_{1}, S_{2}, \ldots, S_{m}\right) . j_{\text {th }}$ attribute of $i_{\text {th }}$ node is defined as $F_{i}\left(S_{j}\right)(i=1,2, \ldots, n ; j=1,2, \ldots, m)$. The decision matrix is as follows:

$$
E=\left(\begin{array}{ccc}
F_{1}\left(S_{1}\right) & \cdots & F_{1}\left(S_{m}\right) \\
\vdots & \ddots & \vdots \\
F_{n}\left(S_{1}\right) & \cdots & F_{n}\left(S_{m}\right)
\end{array}\right)
$$

For comparison, the decision matrix could be optimized by the following normalization processing:

$$
r_{i j}=\frac{F_{i}\left(S_{j}\right)}{\sqrt{\sum_{i=1}^{n} F_{i}\left(S_{j}\right)^{2}}} .
$$

Then the decision matrix could be updated to $R=$ $\left(r_{i j}\right)_{n \times m}$.

Due to the different importance of different regions to the whole network, weight is assigned for each evaluation index to make the algorithm more universal. We denote the $j_{\text {th }}$ index weight as $\omega_{j}(j=1,2, \ldots, m)$, and $\sum_{j=1}^{m} \omega_{j}=1$. Then we denote the weighted normalized matrix as

$$
C=\left(c_{i j}\right)=\left(\omega_{j} r_{i j}\right)=\left(\begin{array}{ccc}
\omega_{1} r_{11} & \cdots & \omega_{m} r_{1 m} \\
\vdots & \ddots & \vdots \\
\omega_{1} r_{n 1} & \cdots & \omega_{m} r_{n m}
\end{array}\right) \text {. }
$$

According to matrix $C$, the positive ideal solution $F^{+}$and the negative ideal solution $F^{-}$are denoted as follows:

$$
\begin{aligned}
& F^{+}=\left\{c_{1}^{+}, c_{2}^{+}, \ldots, c_{m}^{+}\right\}, \\
& F^{-}=\left\{c_{1}^{-}, c_{2}^{-}, \ldots, c_{m}^{-}\right\},
\end{aligned}
$$

where $c_{j}^{+}=\max _{i \in k}\left(c_{i j}\right), c_{j}^{-}=\min _{i \in k}\left(c_{i j}\right)$, and $k=(1,2, \ldots$, $m)$.

We denote the distance from every solution $F_{i}$ to the positive ideal solution $\mathrm{F}^{+}$and the negative ideal solution $\mathrm{F}^{-}$, respectively, denoted by

$$
\begin{aligned}
& D_{i}^{+}=\left\|F_{i}-F^{+}\right\|=\sqrt{\sum_{j=1}^{m}\left(c_{i j}-c_{j}^{+}\right)^{2}}, \\
& D_{i}^{-}=\left\|F_{i}-F^{-}\right\|=\sqrt{\sum_{j=1}^{m}\left(c_{i j}-c_{j}^{-}\right)^{2}} .
\end{aligned}
$$

Then we calculate and sort the ideal solutions similarity degree $Z_{i}$, denoted by

$$
Z_{i}=\frac{D_{i}^{-}}{\left(D_{i}^{-}+D_{i}^{+}\right)} .
$$

According to the TOPSIS method, the node with maximum $Z_{i}$ is the suspected critical node.

4.2. Algorithm Process. It is assumed that OSN has $n$ Ferry nodes and $m$ regions. TOPSIS based synthetic evaluation of region contributions algorithm can be described as follows:

(1) At first, denote the length of time as $\Delta T$. With the sampling analysis on the data of Sink node, the normalization matrix $R=\left(r_{i j}\right)$ can be figured out by (2).

(2) Construct weighted normalized matrix $C$ by (2), (3), and (4).

(3) Determine the positive ideal solution $F^{+}$and the negative ideal solution $F^{-}$by (5a) and (5b).

(4) Calculate the distance from every solution $F_{i}$ to the positive ideal solution $F^{+}$and the negative ideal solution $F^{-}$by (6a) and (6b).

(5) Calculate the similarity degree between each solution and ideal solution by (7). The node with maximum $Z_{i}$ is the suspected critical node.

(6) Repeat the above steps and denote the length of prediction time as $N \cdot \Delta T$. According to (1), the emergence probability of each node can be recorded so that the node with maximum $\left(P_{s}\right)$ is the critical node of the network.

\section{Experiments and Analysis}

5.1. Experimental Scenarios and Related Parameters. As is shown in Figures 3-6, four typical scenarios are simulated on the Opportunistic Networking Environment (ONE). 


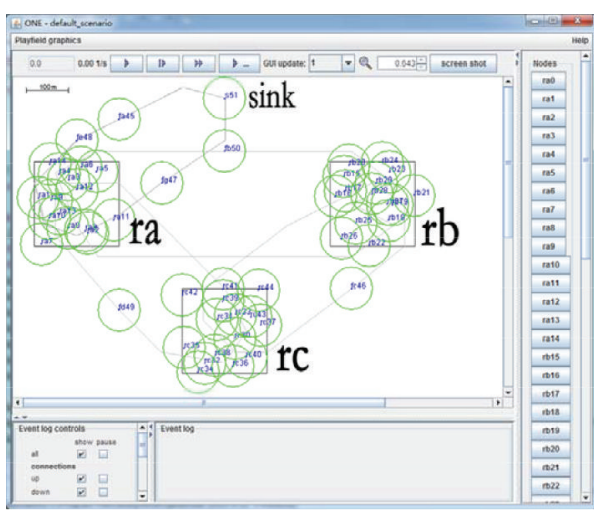

(a) Simulation

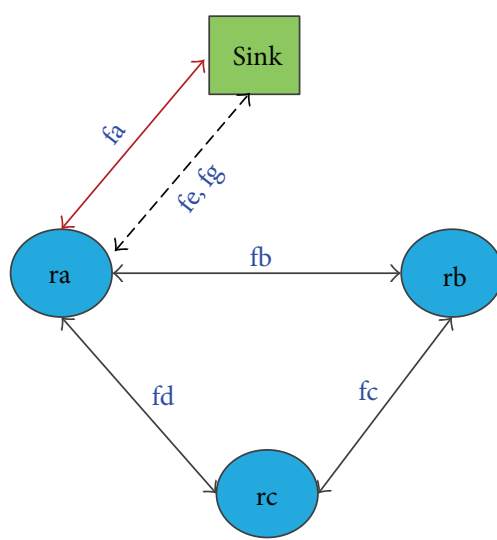

$\begin{array}{ll}\leftrightarrow \text { Messages } & \triangle \text { Ferry node } \\ \bigcirc \text { Region node } & \square \text { Sink node }\end{array}$

(b) Model

Figure 3: Scenario A.

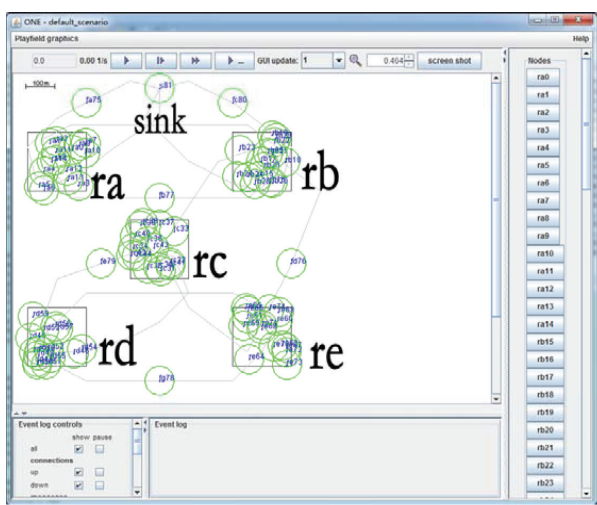

(a) Simulation

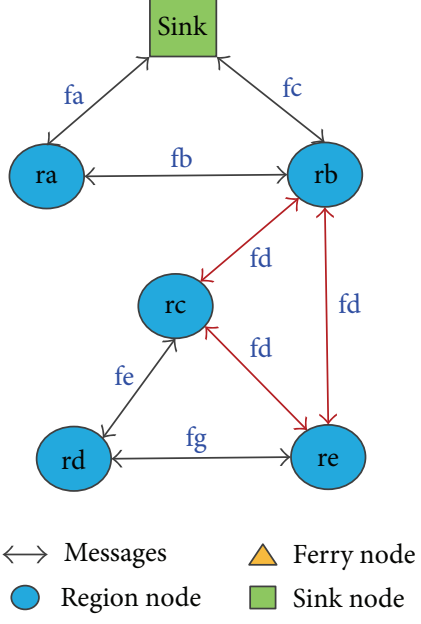

(b) Model

FIgURE 4: Scenario B.

In Figure 3, there is no cut-vertex in Scenario A and there are three region nodes including $\mathrm{ra}, \mathrm{rb}$, and $\mathrm{rc}$ and four Ferry nodes including $\mathrm{fa}, \mathrm{fb}, \mathrm{fc}$, and $\mathrm{fd}$. Among the Ferry nodes, $\mathrm{fb}, \mathrm{fc}$, and fd only provide the communication opportunities among the regions of ra, rb, and rc. However, Ferry nodes fa, $\mathrm{fe}$, and fg provide the communication opportunities between region ra and Sink node which lead to the connection of the whole network. In addition, the black thick lines show that most of the communication opportunities of region ra are provided by Ferry node fa and the dotted lines show that the communication opportunities supported by fe and fg between ra and Sink node are very few, which indicates that node $\mathrm{fa}$ is the critical node of the network in Scenario A.

In Figure 4, Scenario B shows that node fd is the critical node. If node $\mathrm{fd}$ is failure or removed, regions $\mathrm{rc}, \mathrm{rd}$, and re cannot communicate to Sink node anymore. The network split occurs so that node fd is the cut-vertex as well as the critical node of the network.

In some more complex situations like Scenario $\mathrm{C}$ and Scenario D, in Figure 5, there is no cut-vertex in Scenario C and most of the communication opportunities among regions $\mathrm{ra}$ and $\mathrm{rb}$ and Sink node are provided by node fa; thus it can be seen that $\mathrm{fa}$ is the critical node of the network in Scenario C.

In Figure 6, there are several cut-vertexes in Scenario D such as nodes fc, fd, and fe and they are the critical nodes as well.

5.2. Results Analysis. We have made experiments 100 times for each scenario. Table 1 shows the experimental results after the statistical analysis of experimental data.

As shown in Table 1, the maximum appearance possibility $\max \left(P_{s}\right)$ is $0.33 \%$ in Scenario A and $0.44 \%$ in Scenario C. Meanwhile, the corresponding prediction results are both fa. 
TABLE 1: Experiment results.

\begin{tabular}{|c|c|c|c|c|c|c|c|c|c|}
\hline \multirow{2}{*}{ Scenario } & \multirow{2}{*}{$\mathrm{CN}$} & \multicolumn{6}{|c|}{ Appearance possibility $P_{s}(\%)$} & \multirow{2}{*}{$\max \left(P_{s}\right)(\%)$} & \multirow{2}{*}{ Result } \\
\hline & & $\mathrm{fa}$ & $\mathrm{fb}$ & $\mathrm{fc}$ & $\mathrm{fd}$ & $\mathrm{fe}$ & fg & & \\
\hline Scenario A & fa & 0.33 & 0.04 & 0 & 0.06 & 0.03 & 0.06 & 0.33 & $\mathrm{fa}$ \\
\hline Scenario B & $\mathrm{fd}$ & 0.31 & 0 & 0.35 & 0.52 & 0.02 & 0 & 0.52 & $\mathrm{fd}$ \\
\hline Scenario C & $\mathrm{fa}$ & 0.44 & 0.02 & 0.24 & 0.01 & 0 & 0 & 0.44 & $\mathrm{fa}$ \\
\hline Scenario D & $\mathrm{fc}, \mathrm{fd}, \mathrm{fe}$ & 0.22 & 0.17 & 0.52 & 0.52 & 0.52 & - & 0.52 & $\mathrm{fc}, \mathrm{fd}, \mathrm{fe}$ \\
\hline
\end{tabular}

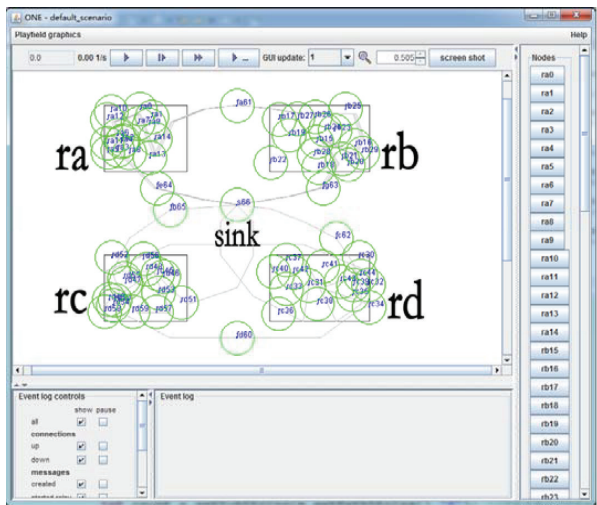

(a) Simulation

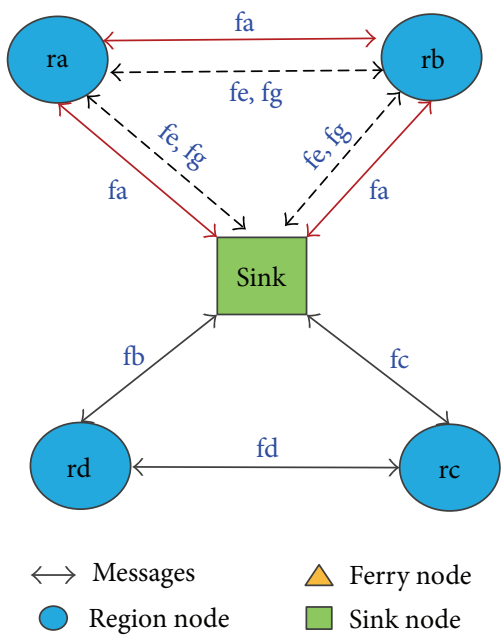

(b) Model

Figure 5: Scenario C.

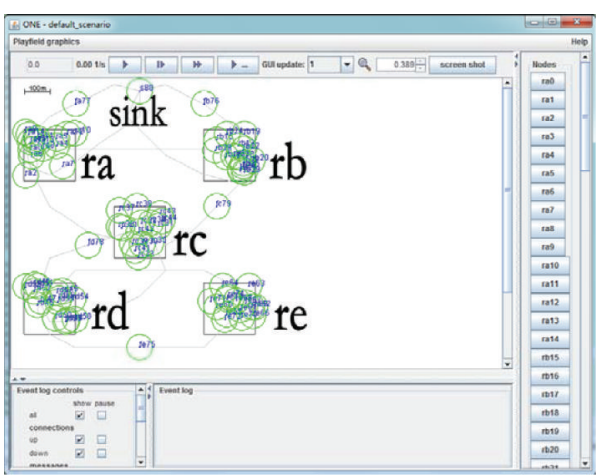

(a) Simulation

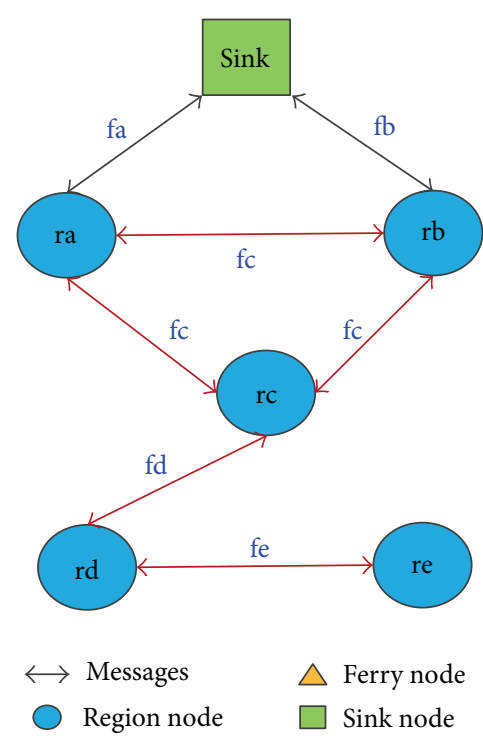

(b) Model

Figure 6: Scenario D. 
In Scenario B and Scenario D, the max appearance possibility $\max \left(P_{s}\right)$ is for both $0.52 \%$. The prediction result in Scenario B is $\mathrm{fd}$ and the results in Scenario D are $\mathrm{fc}, \mathrm{fd}$, and fe. Thus it can be seen that the predicted results of these four scenarios are completely the same with the actual value mentioned above. Therefore, our algorithm can well predict the critical nodes of OSN.

\section{Conclusions}

Considering the dynamic of OSN, this paper proposed a MADM based method to predict critical nodes. First, the region contribution is introduced to present the dependency of regions on Ferry nodes. Then, the comprehensive region contributions are estimated by the MADM method. At last, the experimental results show that, for different OSN scenarios, our method can predict the critical nodes of the network effectively.

\section{Competing Interests}

The authors declare no competing interests.

\section{Acknowledgments}

This work is supported in part by grants from the National Natural Science Foundation of China (nos. 61262020, 61363015, 61501218, and 61501217).

\section{References}

[1] H.-D. Ma, P.-Y. Yuan, and D. Zhao, "Research progress on routing problem in mobile opportunistic networks," Journal of Software, vol. 26, no. 3, pp. 600-616, 2015.

[2] Z. Ma, Y. Sun, and T. Mei, "Survey on wireless sensors network," Journal on Communications, vol. 25, no. 4, pp. 114-124, 2004.

[3] J. C. Nacher and T. Akutsu, "Analysis on critical nodes in controlling complex networks using dominating sets," in Proceedings of the 9th International Conference on Signal-Image Technology and Internet-Based Systems (SITIS '13), pp. 649-654, IEEE, Kyoto, Japan, December 2013.

[4] G. Du, L. He, and J. Fang, "The component importance evaluation of power converter based on complex network," in Proceedings of the International Power Electronics and Application Conference and Exposition, pp. 988-992, IEEE, Shanghai, China, November 2014.

[5] J. Lin, F. Dai, B. Li et al., "Electromagnetic compatibility supernetwork modeling and node importance evaluation," in Proceedings of the 5th International Conference on Intelligent Human-Machine Systems and Cybernetics (IHMSC '13), pp. 306-310, Hangzhou, China, August 2013.

[6] L. Le and Y. Hewei, "A new method for evaluating node importance in complex networks based on data field theory," in Proceedings of the 1st International Conference on Networking and Distributed Computing (ICNDC '10), pp. 133-136, IEEE, Hangzhou, China, October 2010.

[7] F. Hu, Y. Liu, and J. Jin, "Multi-index evaluation algorithm based on locally linear embedding for the node importance in complex networks," in Proceedings of the IEEE 13th International Symposium on Distributed Computing and Applications to Business, Engineering and Science (DCABES '14), pp. 138-142, Xianning, China, November 2014.

[8] J. Li, Y. Tian, M. Sheng et al., "Split detection for large scale," Ad Hoc Networks, vol. 29, no. 9, pp. 54-61, 2008.

[9] C. Huang, W. Furong, H. Benxiong et al., "Preventing natural and malicious network split in ad hoc networks using cooperative healing cell," in Proceedings of the IEEE International Conference on Communications Workshops (ICC Workshops '08), pp. 158-163, Beijing, China, 2008.

[10] S. Xiong and J. Li, "An efficient algorithm for cut vertex detection in wireless sensor networks," in Proceedings of the IEEE 30th International Conference on Distributed Computing Systems, pp. 368-377, Genoa, Italy, June 2010.

[11] R. Lin, B. Wu, Y. Zhao, H. Zou, and L. Liu, "Critical nodes detecting in virtual networking environment," in Proceedings of the IEEE World Congress on Services (SERVICES '14), pp. 317322, IEEE, Anchorage, Alaska, USA, June-July 2014.

[12] D. Zhang and J. P. G. Sterbenz, "Analysis of critical node attacks in mobile ad hoc networks," in Proceedings of the 6th International Workshop on Reliable Networks Design and Modeling (RNDM '14), pp. 171-178, IEEE, Barcelona, Spain, November 2014.

[13] Y. Shen, N. T. Dinh, and M. T. Thai, "Adaptive algorithms for detecting critical links and nodes in dynamic networks," in Proceedings of the Military Communications Conference (MILCOM '12), pp. 1-6, IEEE, Orlando, Fla, USA, November 2012.

[14] G. Ausiello, D. Firmani, and L. Laura, "The (betweenness) centrality of critical nodes and network cores," in Proceedings of the 9th International Wireless Communications and Mobile Computing Conference (IWCMC '13), pp. 90-95, IEEE, July 2013.

[15] H. W. Corley and D. Y. Sha, "Most vital links and nodes in weighted networks," Operations Research Letters, vol. 1, no. 4, pp. 157-160, 1982.

[16] Y. Chen, A. Hu, and X. Hu, "Evaluation method for node importance in communication networks," Journal on Communications, vol. 25, no. 8, pp. 129-134, 2004.

[17] W. Xiao, W. Tan, B. Ge, and F. Li, "Fast method for node importance evaluation in network," Systems Engineering-Theory \& Practice, vol. 33, no. 7, pp. 1898-1904, 2013.

[18] D. Goyal and J. Caffery, "Partitioning avoidance in mobile ad hoc networks using network survivability concepts," in Proceedings of the 7th International Symposium on Computers and Communications (ISCC '02), pp. 553-558, July 2002.

[19] J. Hu, B. Wang, and D. Lee, "Evaluating node importance with multi-criteria," in Proceedings of the IEEE/ACM International Conference on Green Computing and Communications (GreenCom '10), 2010 IEEE/ACM International Conference on Cyber, Physical and Social Computing (CPSCom '10), pp. 792-797, IEEE Computer Society, Hangzhou, China, December 2010.

[20] B. Liu, W. Wang, Y. Li et al., "Crucial node decision algorithm based on energy in WSN," Journal of Electronics and Information Technology, vol. 36, no. 7, pp. 1728-1734, 2014.

[21] X. Zhou, F.-M. Zhang, K.-W. Li, X.-B. Hui, and H.-S. Wu, "Finding vital node by node importance evaluation matrix in complex networks," Acta Physica Sinica, vol. 61, no. 5, Article ID 050201, 2012.

[22] W. Fan and Z. Liu, "Ranking method for node importance based on efficiency matrix," Journal of Southwest Jiaotong University, vol. 49, no. 2, pp. 337-342, 2014.

[23] M. E. Newman, Networks: An Introduction, Oxford University Press, Oxford, UK, 2010. 
[24] J.-W. Wang, L.-L. Rong, and T.-Z. Guo, "A new measure method of network node importance based on local characteristics," Journal of Dalian University of Technology, vol. 50, no. 5, pp. 822-826, 2010.

[25] K.-I. Goh, E. Oh, B. Kahng, and D. Kim, "Betweenness centrality correlation in social networks," Physical Review E-Statistical, Nonlinear, and Soft Matter Physics, vol. 67, no. 1, Article ID 017101, 2003.

[26] A. Budanitsky and G. Hirst, "Evaluating WordNet-based measures of lexical semantic relatedness," Comutational Linguistics, vol. 32, no. 1, pp. 13-47, 2006.

[27] R. Burke, "Semantic ratings and heuristic similarity for collaborative filtering," in Proceedings of the AAAI Workshop on Knowledge-Based Electronic Markets, Austin, Tex, USA, July 2000.

[28] G. Ausiello, D. Firmani, and L. Laura, "The (betweenness) centrality of critical nodes and network cores," in Proceedings of the IEEE 9th International Sardinia Wireless Communications and Mobile Computing Conference (IWCMC '13), pp. 90-95, Sardinia, Italy, July 2013.

[29] J. K. Lou, S. D. Lin, K. T. Chen, and C. L. Lei, "What can the temporal social behavior tell us? An estimation of vertexbetweenness using dynamic social information," in Proceedings of the International Conference on Advances in Social Networks Analysis and Mining (ASONAM '10), pp. 56-63, IEEE, Odense, Denmark, August 2010.

[30] D. Chen, L. Lü, M.-S. Shang, Y.-C. Zhang, and T. Zhou, "Identifying influential nodes in complex networks," Physica A: Statistical Mechanics and Its Applications, vol. 391, no. 4, pp. 1777-1787, 2012.

[31] Z. Ren, F. Shao, J. Liu et al., "Node importance measurement based on the degree and clustering coefficient information," Acta Physica Sinica, vol. 62, no. 12, pp. 522-526, 2013.

[32] D. T. Nguyen, Y. Shen, and M. T. Thai, "Detecting critical nodes in interdependent power networks for vulnerability assessment," IEEE Transactions on Smart Grid, vol. 4, no. 1, pp. 151-159, 2013. 


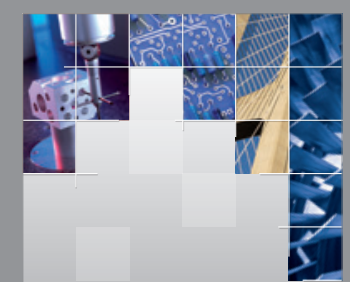

\section{Enfincering}
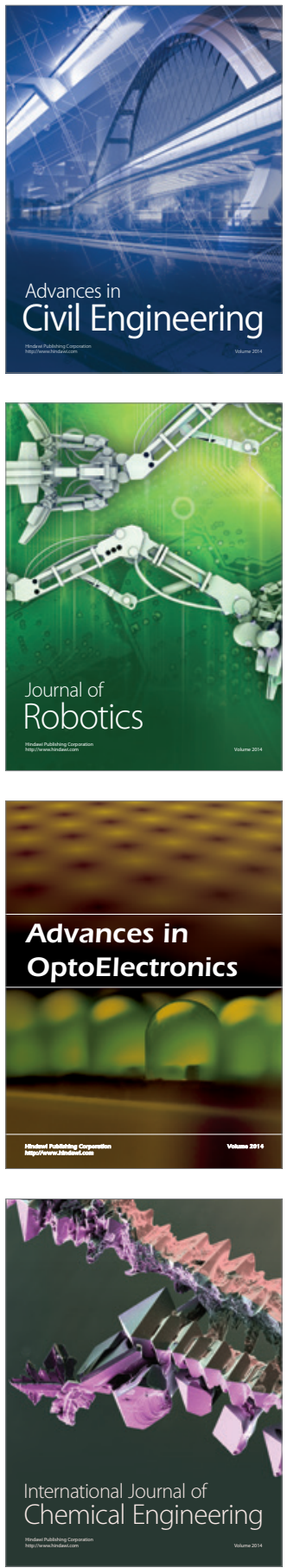

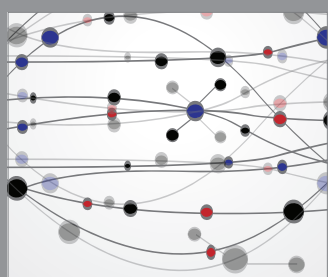

The Scientific World Journal

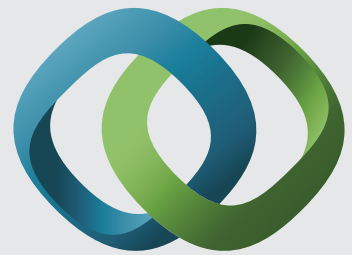

\section{Hindawi}

Submit your manuscripts at

http://www.hindawi.com
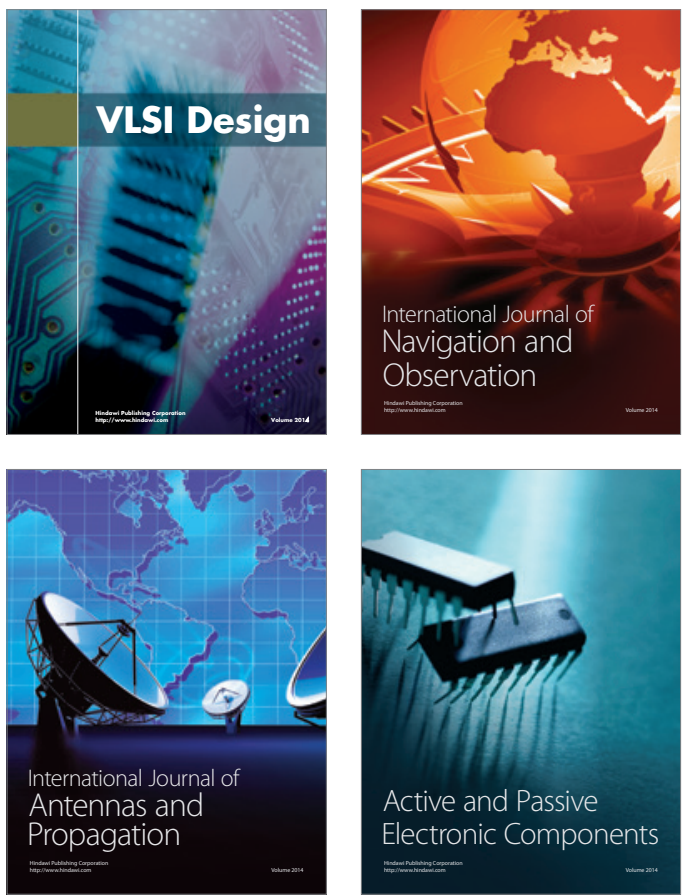
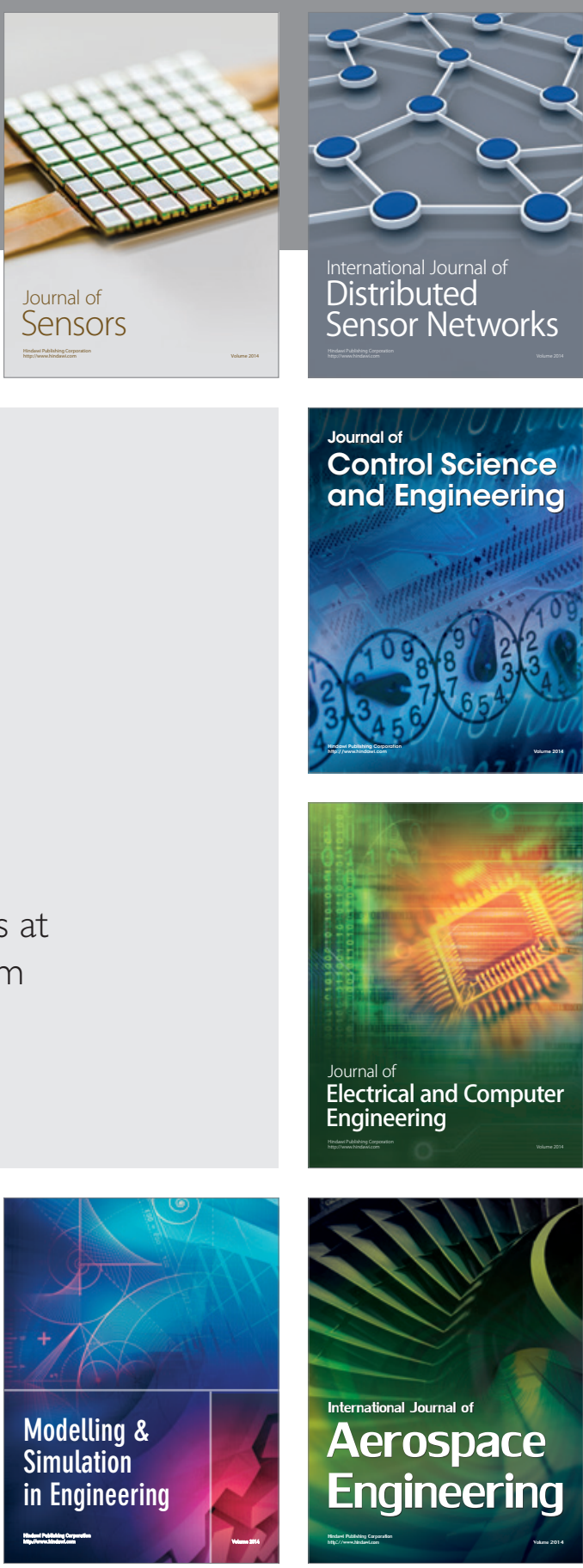

International Journal of

Distributed

Sensor Networks

Journal of

Control Science

and Engineering
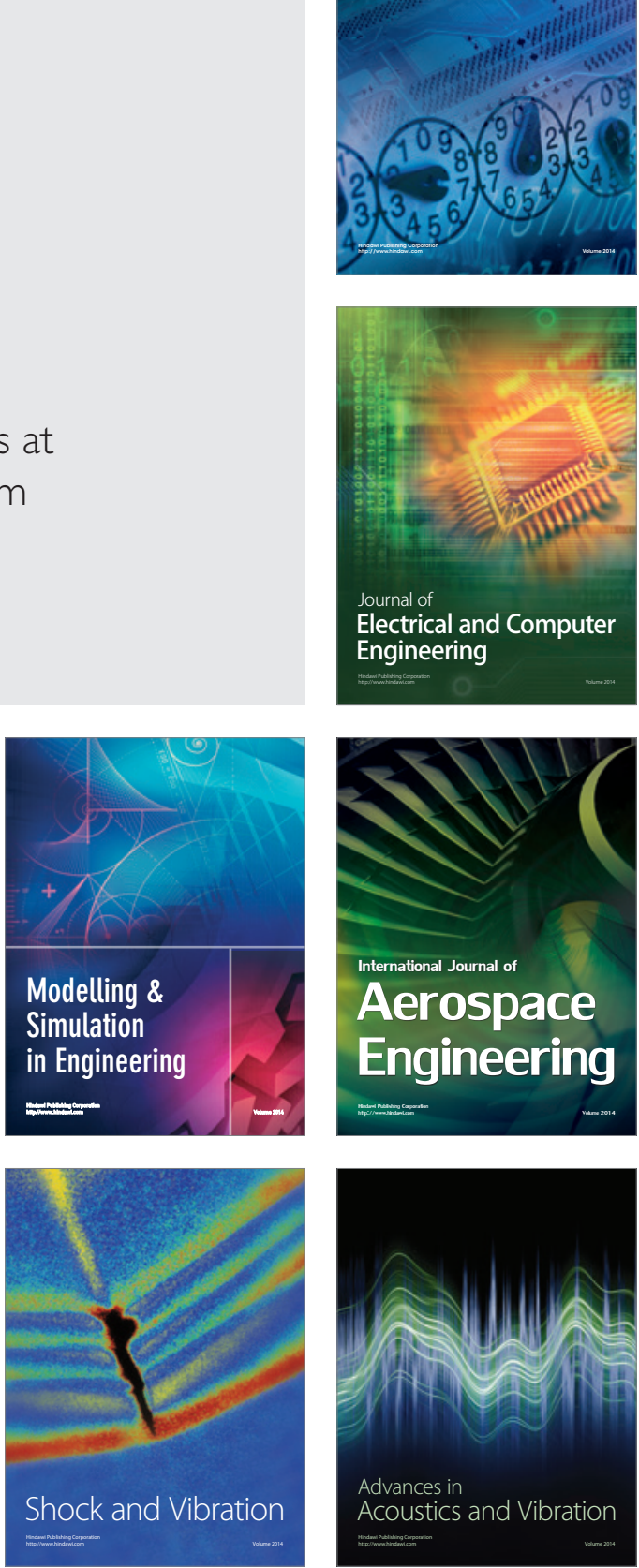